\title{
Simulation Analysis of OLSR and Its Variant with Cooperative MPR Selection on NS-2.35 in Mobile Ad-Hoc Networks
}

\author{
Shelja Sharma, Suresh Kumar \\ Department of Computer Science \& Engineering, Manav Rachna International Institute of Research and Studies \\ (formerly MRIU), Faridabad, Haryana, India \\ E-mail Ids: shelja.fet@mriu.edu.in, suresh.fet@mriu.edu.in
}

Received: 28 December 2017; Accepted: 16 April 2018; Published: 08 July 2018

\begin{abstract}
Mobile Ad-Hoc networks (MANETs) can be classified as Decentralized, Independent and SelfOrganizing dynamic networks of intellectual movable nodes. In such networks, devices are connected by provisional wireless links. Dynamic topology imposes challenges in developing an efficient routing protocol, for enabling successful communication between mobile devices. Based on the nature of working, proactive \& reactive protocols are the two broadly classified categories of routing protocols. This paper presents, relative experimental analysis of proactive routing protocols viz., Optimized Link State Routing Protocol (OLSR) and its variant (Kenji Yamada et al., 2010) with Cooperative Multi-Point Relay (MPR) Selection. To compare OLSR and its variant protocol, the Network Simulator- 2.35 is used to carry out numerous simulations, on arbitrary scenarios, by varying the number of network nodes \& mobility of nodes. As per the simulation outcomes, the OLSR with a cooperative MPR selection has outperformed the traditional OLSR protocol in static scenarios or when the network load has been varied. On the contrary, the traditional OLSR protocol has performed better in mobile scenarios. But, as demonstrated from various experimentations, it exhibits higher Routing Overheads as compared to its variant protocol. Further, on the basis of simulation results, efforts can be made in the direction of performance optimization of OLSR and its variant protocol, to improve its performance in highly mobile scenarios as well, keeping in view other performance metrics.
\end{abstract}

Index Terms-Wireless Communication, Communication Network System, OLSR, Routing Protocol, NS- 2.35, MANET.

\section{INTRODUCTION}

Mobile Ad-Hoc network is a group of self- organizing portable devices, characterized by dynamic topology, distributed routing, multi-hop wireless links and ease of deployment. These characteristics impose new demands on the routing protocols. Routing is a complex task, as nodes can leave and join the network arbitrarily, due to dynamic nature.

Based upon working Principle, the routing protocols for MANETs may be grouped as a Table-driven, Reactive and Hybrid routing protocols [5]. The protocols, which rely on consistently maintaining the topology information, proactively are called Table-Driven or Proactive protocols. Examples of proactive protocols include CGSR [1], DSDV [7], WRP [9] etc. Whereas, On-Demand or Reactive routing schemes minimize the control overheads, by creating routes only when they are required. Few examples of Reactive protocols include TORA [6], DSR [2, 4], ABR [10] and AODV [8]. The combination of Table-Driven \& On-Demand protocols result in Hybrid protocols, SLURP [1] and ZRP [3] are examples of hybrid protocols.

Considering the Proactive category, the DestinationSequenced Distance Vector (DSDV) is an example of a table-driven routing scheme and employs table for maintaining prior routing information. Each node keeps the record of distance vector to every other router present in the network. Every node or router contributes to propagate control information updates, required for routing. Similarly, the Optimized Link State Routing (OLSR) follows a table driven approach proactively, in which every node keeps track of link status information with neighbors and unlike DSDV protocol, some elected nodes contribute in propagating the control information, called Multi-Point Relay (MPR) nodes.

Due to a proactive approach, to maintain fresh information about routes, table-driven routing strategies suffer from the problem of higher control or routing overheads. The OLSR protocol was developed, as an effort to diminish control traffic [11, 12], utilizing only some selected neighbor links for propagating control information, unlike DSDV proactive protocol [13, 14]. Although, the logic of flooding control information has been modified in OLSR protocol for minimizing control traffic, as compared to other routing protocols, but experimental results revealed that the OLSR protocol exhibits still comparatively high routing overhead as shown in our previous publication [24] and references 
$[17,18,28]$. So, an attempt has been made by Kenji Yamada et al. [15], to further diminish the control traffic in OLSR protocol with Cooperative MPR selection. They claimed that there is a redundancy in MPR selection procedure of original OLSR protocol. As per their proposed protocol, whenever there are two or more MPR sets for any node, then the single MPR set is selected, based upon maximum no. of shared MPR nodes with MPR sets of neighbor nodes. As per their simulation results, there is a $15 \%$ reduction in Transmission control packets with respect to static scenarios and up to $14 \%$ reduction in mobile scenarios.

We intended to analyze the comparative performance of OLSR (Optimized Link State Routing) and its variant [15] with Cooperative MPR selection. The NS-2.35 is chosen for simulation and analysis. Unlike DSDV protocol, the OLSR protocol is unavailable with the NS2.35 installation. So, the OLSR protocol and its variant [15] with Cooperative MPR selection, were patched \& installed on NS- 2.35. In contemplation of evaluating the performance of the above-said protocols, the following performance metrics are considered viz., Packet Delivery Fraction (PDF), Routing Overhead (RO), Packet Loss (PL) and End-to-End Delay (EED). In order to plot graphs and evaluating performance parameters, average results have been considered, from various simulation runs.

\section{RELATED WORKS}

This section presents the work carried out by several authors, in the interest of performance improvement of proactive protocols, specifically the OLSR protocol and also summarizes the conclusion of various experimentations done.

Jia Lu et al. [23], have proposed modifications to the original DSDV protocol, to address its Invalid route repairing problem. Their proposed protocol does not need any fresh information exchange for its invalid route regeneration, but each node maintains two routing tables. As per their simulation results, the packet delivery fraction is improved.

P. Jacquet et al. [11], designed \& developed an OLSR routing protocol destined for mobile Ad-Hoc networks. It's considered as an improvement over a traditional link state protocol. It employs the technique, known as multipoint relaying for efficiently and economically flooding the control information. The authors claimed that their proposed protocol should best suit to denser \& large Ad-Hoc Networks.

Deepali Arora et al. [17], based upon simulation results claimed that the OLSR does not achieve the desired performance in larger \& dense multi-hop mobile networks. They notified, the reason behind is excessive overheads, although, it performed well in the scenario, where maximum nodes are almost stationary. Also, their results diverged from previous claims $[11,18,27]$ that, the OLSR is specifically appropriate for larger \& denser ad-hoc networks.

S. Mohapatra et al. [18], based upon their experimental outcomes, concluded that, as compared to other tabledriven protocols, the OLSR protocol is a superior option for highly mobile networks, if an area of the network is greater than $600 \mathrm{~m} \times 600 \mathrm{~m}$ and if, the applicationoriented metrics viz. PDF and Throughput are the main criteria. But, as per their simulation results, the control overhead of DSDV is lower than OLSR protocol.

S. Mohapatra et al. [19], in their subsequent publication, performed a comparative assessment of AODV, DSDV, and OLSR routing protocol using NS- 2. They have analyzed- throughput, packet delivery ratio \& control overhead and concluded that the OLSR gives better packet delivery fraction.

Pore Ghee Lye and John C. McEachen [28], have presented a comparative investigation of DSDV, OLSR, and AODV routing protocols. As per their simulation results, AODV outperforms DSDV and OLSR upon varying mobility rate. On the other hand, OLSR also performs well relatively, but an associated high routing overhead is the constraint in choosing it.

Zheng Yihui et al. [16], have suggested an improved OLSR routing protocol- N3S-OLSR. Unlike OLSR, it additionally utilizes self- sensing information \& hop count to calculate Multi Point Relay sets. Every node maintains sensing information using two modules: Node Status Information and Link Status Information. In order to achieve optimal network performance, their proposed algorithm reduced the probability of choosing weak nodes with poor processing capacity \& poor link properties as an MPR node. The authors concluded that as compared to traditional OLSR protocol, the N3SOLSR improved the packet delivery ratio and prolonged the average path duration.

Yamada Kenji et al. [15], have made efforts to diminish the control overheads of the OLSR protocol. Authors have mentioned that, in traditional OLSR protocol, the process of Multi- Point Relay selection run on every node independently of its neighbors and consequently, exhibits redundancy. They observed that in denser networks, redundant control messages can be included in a single control packet. As per the simulation results, their proposed modification in MPR selection algorithm decreased the no. of Topology Control (TC) messages in OLSR protocol in dense networks.

Yamada Kenji et al. [20], in their subsequent publication, presented a non-distributed algorithm for Multi-Point Relay node selection, aimed at minimizing the no. of TC message senders in the network. However, due to its centralized approach, it cannot be implemented in the OLSR protocol. 
Teruaki Kitasuka et al. [21], have proposed a centralized Multipoint Relay selection algorithm to show, chances of selecting same nodes as MPRs as of neighbors. They calculated the Multipoint Relay ratio and measured the Routing Overheads due to TC messages.

Leonardo Maccari et al. [22], emphasized that, in order to reduce control overheads in OLSR protocol, the main aim should be to reduce the global MPRs in the network rather than diminishing the number of MPRs an individual node selects for itself. The authors have proposed two modifications to the MPR selection criterion. As per their proposed strategy, while selecting MPR nodes, higher priority is given to those nodes, which are already being chosen by some other nodes.

\section{Performance Evaluation}

This section presents the experimental set up for performing simulations. The various simulation parameters considered are listed in a Table 1, for evaluating the performance of OLSR and its variant protocol. Also, the analysis of results has been presented in accordance with the simulation outcomes.

\section{A. Simulation Setup \& Method of Evaluation}

The discrete event simulator- NS- 2.35 is utilized for simulating and evaluating parameters. The NS- 2.35 installation includes only basic protocols like DSDV, AODV \& DSR, but OLSR is unavailable with it. We have considered the software called "UM- OLSR", developed by the University of Murcia, Spain [26] and used the patch- "um-olsr-1.0.tgz", in order to patch \& install OLSR and its variant [15] with Cooperative MPR selection on NS-2.35.

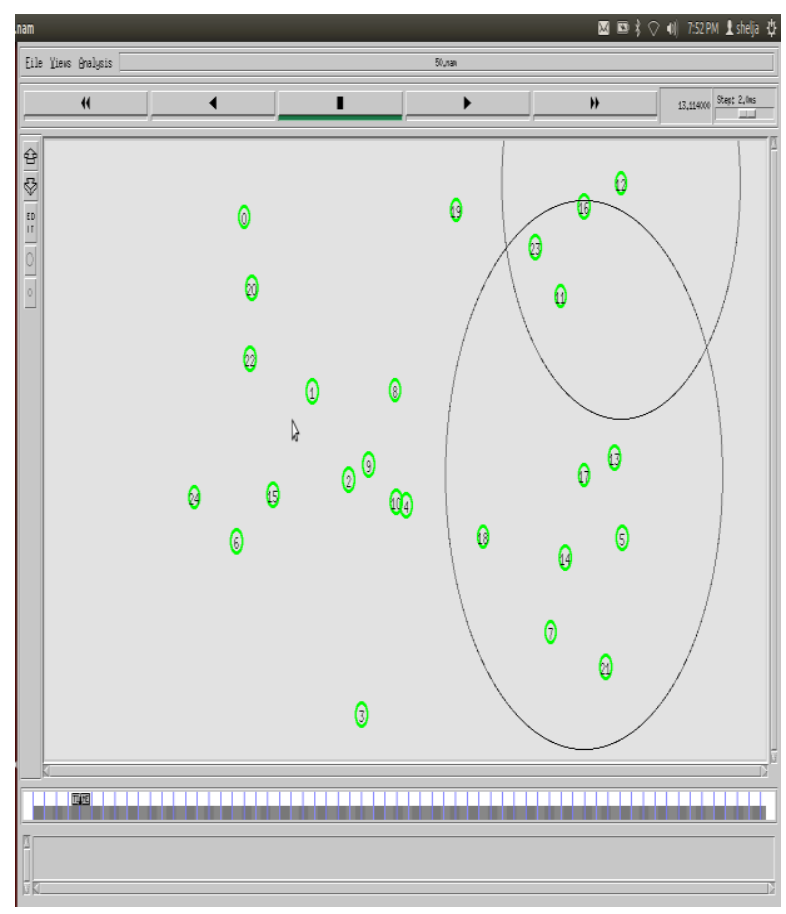

Fig.1. Network scenario during Simulation Process
To generate twenty-five random scenario patterns, pause time is ranged from 0 (s) to 200 (s) and the number of nodes have been varied from 15-35, for generating Constant Bit Rate (CBR) traffic patterns as well. The output produced by NS- 2 simulator is recorded in two files, one is used for calculating \& evaluating the results known as "trace file" and another is used for graphical interpretation, generated by NAM (Network Animator) called the "NAM file". Fig. 1, shows the network scenario at a particular instant during simulation.

We have a varied mobility of nodes \& network load, for carrying simulations several times on arbitrary scenario patterns. The several performance parameters viz., Routing Overhead, End-to-End delay, Packet Delivery Fraction and Packet Loss have been evaluated considering, the average value of the results from various simulation runs.

To evaluate the network performance using OLSR and its variant [15] with Cooperative MPR selection, two cases have been considered. The first case considers the network load analysis, at pause time- 100 (s) and the number of nodes have been varied from 15-35 nodes. Whereas, the second case considers the mobility analysis, with a constant number of nodes- 25 and the pause time has been varied from 0 (s) to 300 (s). Table I, presents the simulation parameters considered.

Table 1. Simulation Prerequisites

\begin{tabular}{|c|l|l|}
\hline S. No & Parameters & Value \\
\hline 1 & Routing Protocols & $\begin{array}{l}\text { OLSR and its variant } \\
\text { with Cooperative MPR } \\
\text { Selection }\end{array}$ \\
\hline 2 & Simulation Duration & $200 \mathrm{~s}$ \\
\hline 3 & MAC Layer & IEEE $802.11 \mathrm{~b}$ \\
\hline 4 & Transmission Range & $250 \mathrm{~m}$ \\
\hline 5 & Simulation Area & $800 \mathrm{~m} \times 500 \mathrm{~m}$ \\
\hline 6 & No. of Nodes & $15,20,25,30,35$ \\
\hline 7 & Node Movement Pattern & Random Waypoint \\
\hline 8 & $\begin{array}{l}\text { Application or Connection } \\
\text { Type }\end{array}$ & Constant Bit Rate $(\mathrm{CBR})$ \\
\hline 9 & Pause Time & $0 \mathrm{~s}, 50 \mathrm{~s}, 100 \mathrm{~s}, 150 \mathrm{~s}, 200 \mathrm{~s}$ \\
\hline 10 & Node Velocity & $20 \mathrm{~m} / \mathrm{s}$ \\
\hline 11 & Maximum Connections & 10 \\
\hline 12 & Size of Packet & $512 \mathrm{bytes}$ \\
\hline 13 & $\begin{array}{l}\text { Rate of Packet } \\
\text { Transmission }\end{array}$ & 4 packets/sec \\
\hline & &
\end{tabular}

\section{B. Simulation Outcomes \& Analysis}

To inspect the performance of OLSR \& its variant [15] with Cooperative MPR selection, this section presents various simulation outcomes and their analysis. Four performance metrics have been considered viz., PDF, PL, RO, and EED. Two cases have been considered, to inspect the network performance. The first case illustrates the Network load analysis and second case demonstrates the Mobility Analysis.

The case I: Network Load Analysis 
In view of this analysis, the simulation duration, pause time and simulation area are fixed at $200(\mathrm{~s}), 100(\mathrm{~s})$ and $800 \times 500\left(\mathrm{~m}^{2}\right)$ respectively, and the no. of nodes have been varied as $15,20,25,30 \& 35$. Whereas, leftover simulation parameters are described in Table I. During simulation, 5 traffic files and 10 random scenarios have been generated. The performance graphs are plotted, considering the average value of results from various simulation runs.

Packet Delivery Fraction: The PDF determines the successful delivery of data packets to respective destinations. The Fig. 2, depicts the Packet Delivery Fraction of the OLSR \& its variant with a Cooperative MPR selection [15]. It is observed from the graph that, the PDF of OLSR is comparatively lower than its variant, irrespective of varying no. of nodes. As the traffic sources increases, when the number of nodes is 35 , there is an increase in PDF of both protocols. Reason behind is, the OLSR with cooperative MPR Selection is based on the concept of selecting an MPR set, which has lager no. of common nodes with MPR sets of neighbors of the corresponding node. It further reduces the no. of TC messages and hence the congestion in the network, resulting in an improved PDF as compared to the OLSR Protocol.

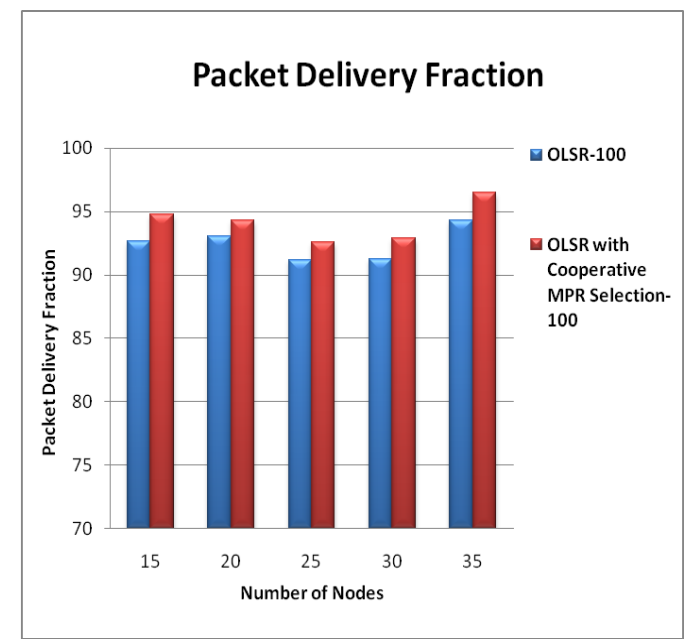

Fig.2. Impact of varying Network Load on Packet Delivery Fraction

Routing Overhead: The routing packets flooded in the network, intended to determine and propagate the information about network routes, account for overheads in the network. As depicted in Fig. 3, the Routing Overhead of the OLSR with Cooperative MPR selection is relatively lower than the original OLSR protocol, irrespective of varying number of nodes. But, as the traffic source increases, both protocols show an increase in routing overhead.

The prominent reason could be the transmission frequency of TC messages and Hello Messages (HM). As the no. of nodes increases, the no. of HM and TC messages increase in the network, intended to keep an up-to-date record of MPR nodes, but resulting in a congested network. Moreover, the HM emission period is preset to 2 sec., and the TC message emission period is preset to $5 \mathrm{sec}$., resulting in higher Routing Overheads in OLSR Protocol experimentally. Whereas, in its variant [15] with Cooperative MPR selection, an effort has been made to reduce the Routing Overhead by modifying the MPR set selection criteria. It is based on the concept of selecting a shared MPR set, in consultation with neighbors. The idea of utilizing common MPR nodes with neighbors, further reduces the no. of TC messages and hence the Routing Overhead in the network.

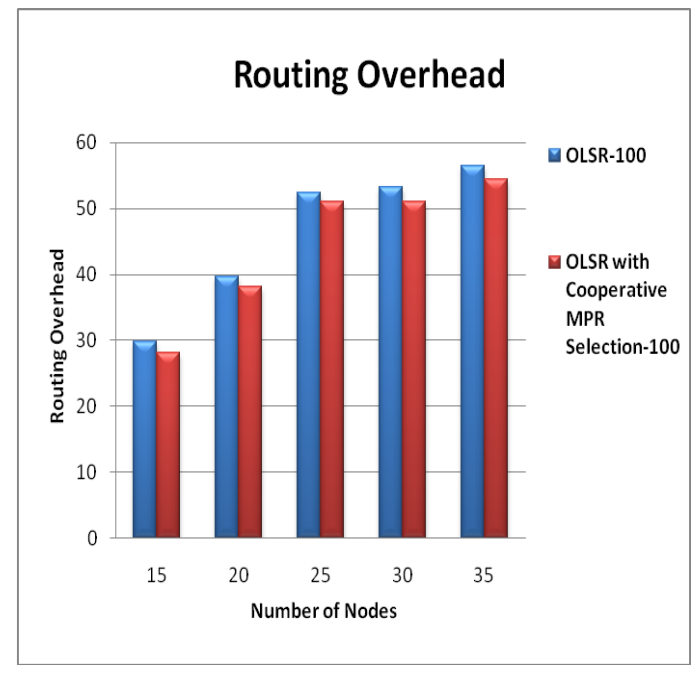

Fig.3. Impact of varying Network Load on Routing Overhead

Packet Loss: Routers may drop packets due to various reasons. Packet Loss measures the total no. of lost packets with respect to transmitted packets. Fig. 4 depicts the number of packets lost, upon varying no. of nodes for the two protocols under consideration. It's perceived from the graph that, the OLSR protocol has relatively lower packet loss rate than its variant [15] protocol at most of the points. As OLSR with a cooperative MPR selection [15], relies on the shared MPR set and consumes more time in re computing the single MPR set based upon common MPR nodes with neighbor nodes, meanwhile the packets may be lost by routers in the absence of updated path, due to mobility.

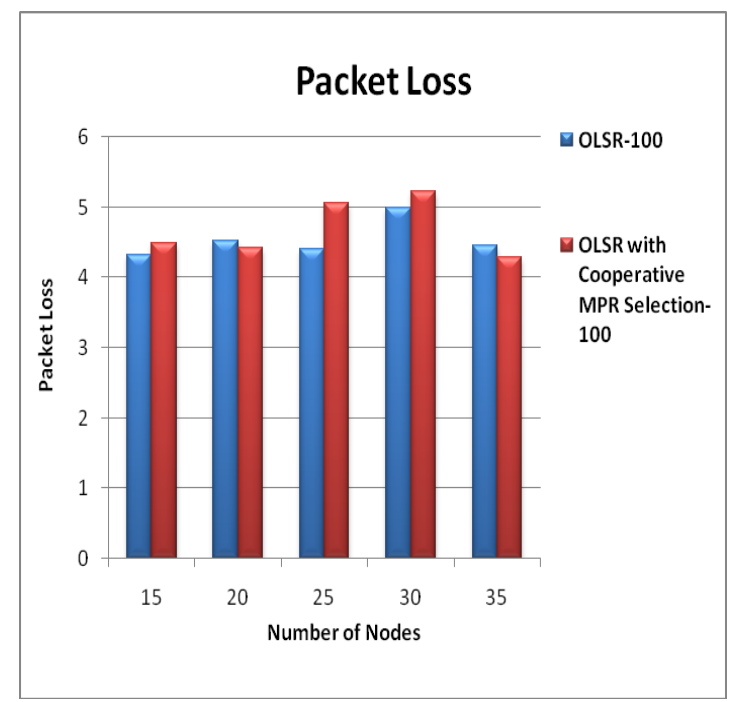

Fig.4. Impact of Varying Network Load on Packet Loss 
End-to-End Delay: It incorporates the entire traveling period incurred by a packet, in order to reach the corresponding destination. Fig. 5 depicts that, the total delay of an individual protocol is varying upon varying the number of nodes. Peculiarly, the EED of OLSR protocol with a cooperative MPR selection [15], is comparatively lower than the traditional OLSR protocol irrespective of varying no. nodes. The reason behind is, an Independent MPR set is utilized by each node in the OLSR protocol, but the shared MPR nodes might be utilized for forwarding TC messages in its variant [15] with cooperative MPR selection, resulting in lower EED comparatively.

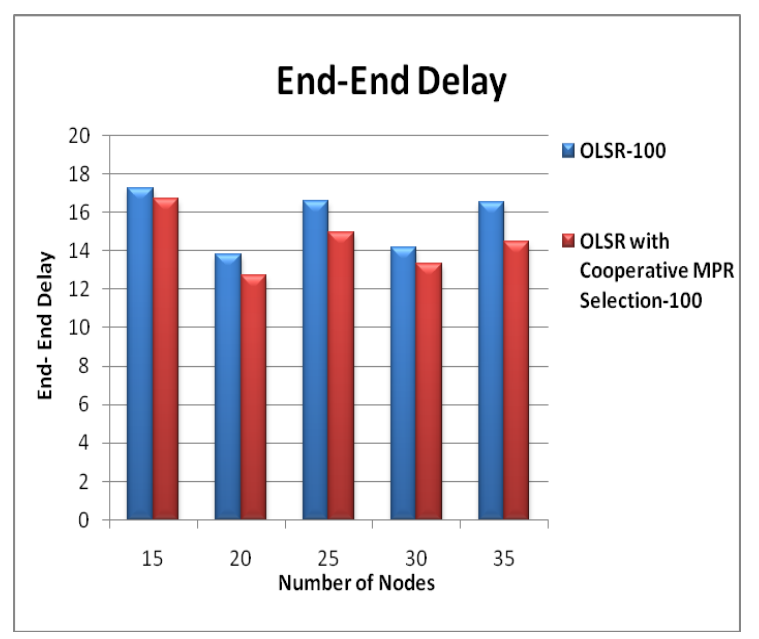

Fig.5. Impact of varying Network Load on End-End Delay

\section{Case II: Mobility Analysis}

In this case, we have considered the constant network size with 20 nodes and pause time is varied from- 0 second to 200 seconds. The consequences of varying mobility have been investigated on the protocol's performance. The connection type used is CBR, with size of packet- 512 bytes and packet transmission rate is preset to 4 packets/ second. The Table I displays leftover simulation parameters.

Packet Delivery Fraction: Fig. 6 depicts the Packet Delivery Fraction of the two protocols with reference to the mobility of nodes. As observed from the graph, the PDF of both protocols is varying upon varying mobility of nodes and it becomes almost nearly $100 \%$ at pause time 200(s). Broadly, the PDF of OLSR and its variant [15] protocol is almost same in all the cases. But, peculiarly the PDF of Yamada's protocol [15] is slightly raised at pause time $100(\mathrm{~s})$, when the network started tending towards the stability, from the state of mobility and thereafter, remains little higher than the OLSR protocol. The reason behind is, the OLSR with a cooperative MPR selection [15], relies on the concept of the shared MPR set and hence further reduces Routing overheads, resulting in an improved PDF comparatively, when the network has turned towards stability.

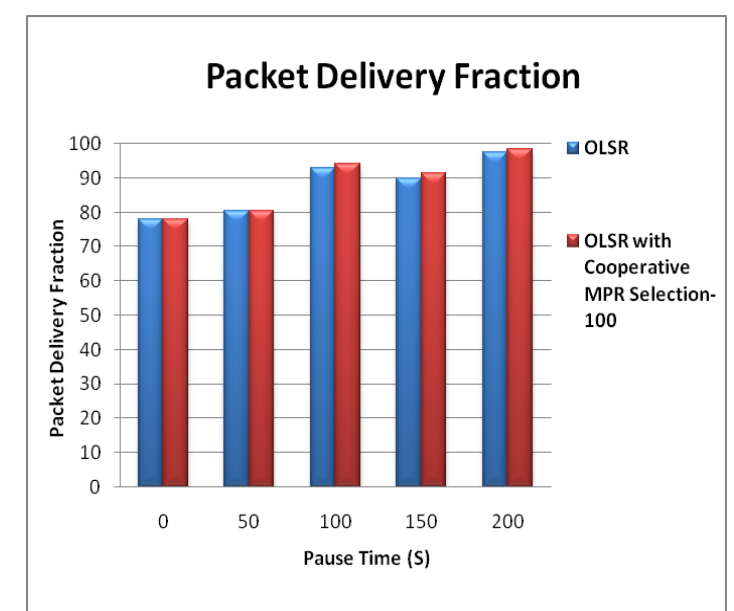

Fig.6. Effect of varying Pause Time on Packet Delivery Fraction

Routing Overhead: The Fig. 7 depicts the Routing Overheads of the two protocols with respect to pause time. As analyzed from the graph, the Yamada's protocol [15], exhibits relatively lower Routing Overhead than the traditional OLSR protocol, regardless of varying mobility of nodes. The control messages are relayed more frequently in traditional OLSR protocol, as the HM emission period is preset to $2 \mathrm{sec}$., and the TC message emission period is preset to $5 \mathrm{sec}$., which results in higher Routing Overheads in OLSR Protocol. Whereas, in Yamada's protocol [15], an effort has been made in reducing the Routing Overheads, by employing the concept of selecting an MPR set, based upon similarity with MPR sets of a neighborhood. The concept of Sharing MPR nodes with neighbors, further reduces no. of TC messages and hence, the Routing Overhead in the network.

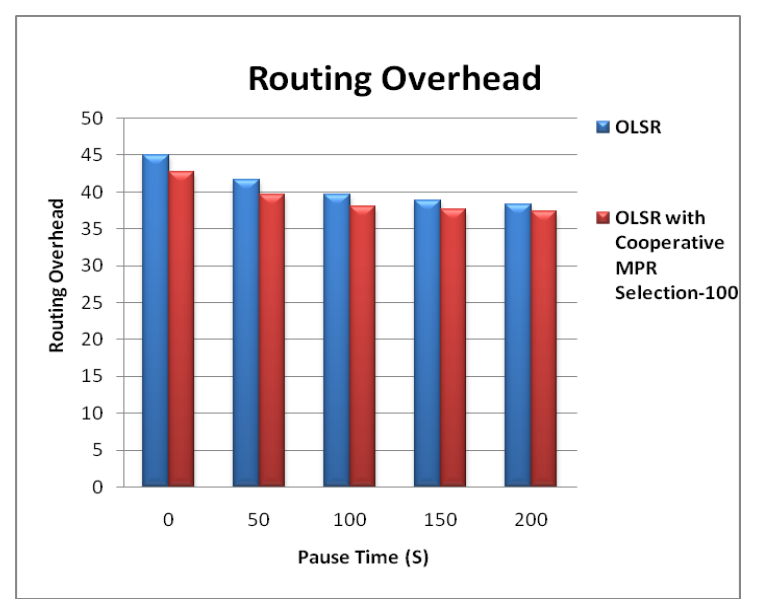

Fig.7. Effect of varying Pause Time on Routing Overhead

Packet Loss: As depicted in Fig. 8, the packet loss of the OLSR is relatively lower than its variant [15] at pause time 0 second and 50 seconds, when the network is mobile. But, when the network is tending towards stability from pause time 100 (s) onwards, the packet loss rate of Yamada's protocol [15] is slightly decreased than the traditional OLSR protocol. 


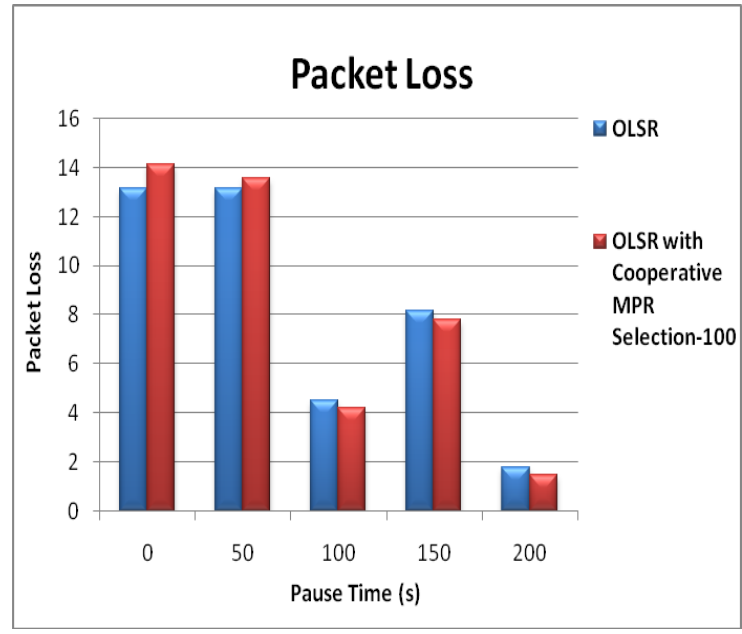

Fig.8. Effect of varying Pause Time on Packet Loss

End-to-End Delay: As depicted in Fig. 9, upon varying mobility of nodes, the total delay of both protocols is fluctuating continually. In particular, the EED of Yamada's protocol [15] is relatively higher than OLSR protocol at pause time 0 second, when the nodes are continually moving. The Yamada's protocol [15] consumes more time in finally reselecting an MPR set based upon common MPR's with neighbor nodes. But, as the network is tending towards stability, the EED of the traditional OLSR protocol is comparatively more than its variant [15].

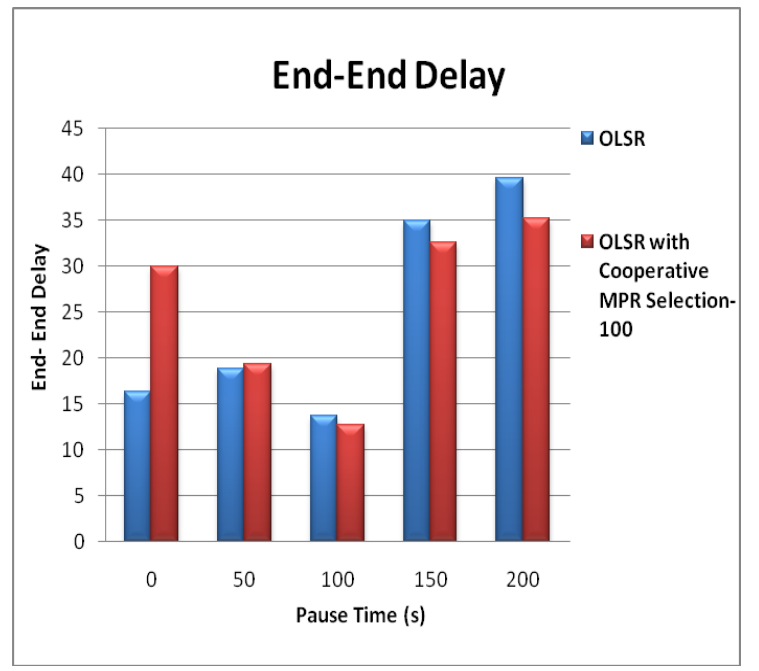

Fig.9. Effect of varying Pause Time on End-End Delay

\section{CONCLUSION}

The OLSR and its variant [15] with cooperative MPR selection, both protocols employ proactive routing strategy, but through different approaches for MPR set selection. As per the literature, proactive protocols exhibit higher control overheads. Aimed at reducing the routing overheads, the OLSR protocol introduced the notion of MPR Nodes. But, experimentations did not reveal the same. One participant reason could be the short transmission intervals for TC message and Hello message transmissions in the OLSR protocol. So, in Yamada's protocol [15] an effort has been made to further reduce the routing overheads, by updating the process of MPR selection. In this paper, an experimental analysis of OLSR and its variant [15] protocol with a cooperative MPR selection has been performed on NS-2.35 simulator. Based upon simulation results, the Yamada's protocol [15] outperformed the traditional OLSR protocol with reference to metrics like Packet Delivery Fraction, when the network load is varied, and the Routing Overhead in all the cases considered, regardless of varying mobility of nodes or network load. But for other metrics, the traditional OLSR has performed comparatively better, in the scenario of highly mobile network, at pause time 0 (s) and 50 (s). On the contrary, when the network load has been varied or the network has turned towards stability, the OLSR protocol with a cooperative MPR selection has performed little better.

Consequently, there is a need to further update the OLSR protocol by employing different routing strategy, which may result into further reduced routing overhead and modifications can also be done in Yamada's protocol [15] to further improve its performance in highly mobile scenarios as well, keeping in view other performance metrics. Hence, in future efforts can be made, Aimed at performance optimization of traditional OLSR and its variant [15] protocol, by working in the direction of further reducing the Routing overheads, as well as improving other performance metrics.

\section{REFERENCES}

[1] Abolhasan Mehran, Wysocki Tadeusz, and Dutkiewicz Eryk, "A review of routing protocols for mobile ad hoc networks", www.elsevier.com/locate/adhoc, Ad Hoc Networks, pp.1-22, 2004.

[2] Broch J., Johnson D. B., and Maltz D. A., "The Dynamic Source Routing Protocol for Mobile Ad Hoc Networks," IETF Internet draft, draft-ietfmanet-dsr-01.txt, (work in progress) Dec. 1998.

[3] Hass Z. J.and Pearlman R.,'ZZone routing protocol for adhoc networks", Internet Draft, draft- ietf-manet-zrp-02.txt, work in progress, 1999.

[4] Johnson David B. and Maltz David A., "Dynamic source routing in ad hoc wireless networks", In Mobile Computing, edited by Tomasz. Imielinski and Hank Korth, chapter 5, pp. 153-181, 1996.

[5] Murthy C. S. R. and Manoj B. S., "Ad Hoc Wireless Networks", Pearson Education, pp. 213-226,2005.

[6] Park Vincent D. and Corson M. Scott., "TemporallyOrdered Routing Algorithm (TORA)", version 1: Functional specification. Internet-Draft, draft-ietf-manettora-spec-00.txt, November 1997.

[7] Perkins Charles E. and Bhagwat Pravin, "Highly Dynamic Destination-Sequenced Distance-Vector Routing (DSDV) for mobile computers", Proc. of SIGCOMM' 94 Conference on Communications Architectures, Protocols and Applications, pp.234-244, August 1994.

[8] Perkins C. E. and Royer E. M., "Ad-hoc On-Demand Distance Vector Routing", Proc. 2nd IEEE Wksp. Mobile Comp. Sys. and Apps., pp. 90-100, Feb. 1999.

[9] Royer E. M. and Toh Chai-Keong, "A review of current routing protocols for ad-hoc mobile wireless networks", 
Proc. IEEE, vol. 6, pp. 46 - 55, April 1999.

[10] Toh C.K,'Long-lived ad-hoc routing based on the concept of associativity", Internet Draft, IETF, March 1999.

[11] Jacquet P., Muhlethaler P., Clausen T., Laouiti A, Qayyum A and Viennot L., "Optimized link state routing protocol for ad hoc networks", In Proceedings of the 5th IEEE Multi-Topic Conference INMIC, pp. 62-68, 2001.

[12] Clausen T. and Jacquet P., "Optimized Link State Routing Protocol (OLSR)", RFC 3626, IETF Network Working Group, available online at https://www.ietf.org/ rfc/rfc3626.txt, October 2003.

[13] Perkins C. E. and Bhagwat P., "Highly Dynamic Destination-Sequenced Distance-Vector Routing (DSDV) for mobile computers", In proceedings of SIGCOMM '94 Conference on Communications Architectures, Protocols and Applications., pp. 234-244, 1994.

[14] Murthy C. S. R. and Manoj B. S.,"Ad Hoc Wireless Networks: Architecture and Protocols", Pearson Education, pp. 330-334, 2005.

[15] Yamada Kenji, Itokawa Tsuyoshi, Kitasuka, and Aritsugi Masayoshi,"Cooperative MPR Selection to Reduce Topology Control Packets in OLSR", TENCON, IEEE proceedings, pp. 293-298, 2010.

[16] ZhengYihui, QueXirong, Wang Wendong, Gong Xiangyang, Ma Jian, "N3S-OLSR: Node-Status SelfSensing Optimized Link-State RoutingProtocols for MANET", International Conference on Communications and Mobile Computing (CMC),IEEE Conference Publications, pp. 288 - 292, 2010.

[17] Deepali Arora, Eamon Millman, Stephen W. Neville, "Assessing the Performance of AODV, DYMO, and OLSR Routing Protocols in the Context of Larger-scale Denser MANETs", IEEE Pacific Rim Conference on Communications, Computers, and Signal Processing, pp. 675-679, 2011.

[18] S. Mohapatra, P. Kanungo, "Performance analysis of AODV, DSR, OLSR and DSDV Routing Protocols using NS2 Simulator", International Conference on Communication Technology and System Design, www.elsevier.com/locate/procedia, pp. 69-76, 2012.

[19] S. Mohapatra, P. Kanungo,"Comparative performance analysis of MANET routing protocols using nS2 simulator", Published in Springer's Computational Intelligence and Information Technology series, pp. 731736, 2011.

[20] Yamada Kenji, Tsuyoshi Itokawa, "Redundant TC Message Senders in OLSR", IEICE TRANSACTIONS on Information and Systems, pp.3269-3272, 2010.

[21] Teruaki Kitasuka, Shigeaki Tagashira,"Density of Multipoint Relays in Dense Wireless Multi-hop Networks", Published in IEEE International Conference on Networking and Computing, pp. 134-140, 2011.

[22] Leonardo Maccari, Renato Lo Cigno,"How to Reduce and Stabilize MPR sets in OLSR networks", In Proceedings of the IEEE International Conference on WiMob, pp. 373-380, USA, 2012.

[23] Jia Lu, Junhui Wang, Gang Han, Baoliang, "A DoubleLinks Scheme for DSDV in Mobile Ad Hoc Networks", Published in Springer's Communications in Computer and Information Science (CCIS) series, pp. 538-547, 2012.

[24] Shelja Sharma, Suresh Kumar, "Experimental Analysis of OLSR and DSDV routing Protocols in Mobile Ad Hoc Networks", Published online in MECS International Journal of Computer Network \& Information Security, pp. 21-29, July 2015.

[25] The network simulator - $\quad$ ns-2, http://www.isi.edu/nsnam/ns/on. The CMU Monarch
Project, Computer Science Department, Carnegie Mellon University, //www.cs.cmu.edu., Accessed on Dec. 2013.

[26] Francisco J. Ros, "um-olsr-1.0.tgz", University of Murcia, Spain, available online at http://masimum.inf.um.es/ fjrm/development/um-olsr/ and http://sourceforge.net/projects/um-olsr/, 2006. Accessed in Jan. 2015.

[27] Rahman M. and Muktadir A. Al, "The impact of data send rate, node velocity and transmission range on QoS parameters of OLSR and DYMO MANET routing protocols", In Proceedings of IEEE 10th international Conference on Computer and Information Technology, ICCIT, pp. 1-6, Dec. 2007.

[28] Pore Ghee Lye and John C. Mc Eachen, "A Comparison of Optimized Link State Routing with Traditional Ad-Hoc Routing Protocols", Edited by Beata J Wysocki\&Tadeusz A Wysocki, Published in 5th Workshop on the Internet, Telecommunications and Signal Processing, Hobart, Australia, pp. 1-7, Dec. 2006.

[29] Suresh Kumar, R K Rathy, D Pandey, "Traffic Pattern Based Performance Analysis of Routing Protocols in Adhoc Networks", Journal of International Academy of Physical Sciences, Vol. 15, No.1, pp. 123-140, ISSN 0974 -9373, 2011.

[30] Suresh Kumar, Suman Kumari, R K Rathy, "Traffic Pattern Based Performance Comparison of AODV, DSDV \& OLSR MANET Routing Protocols using Freeway Mobility Model", International Journal of Computer Science and Information Technologies, Vol. 2 (4), pp. 1606-1611, ISSN : 0975-9646, 2011.

[31] Shelja Sharma, Suresh Kumar, "Performance Improvement of OLSR Protocol by Modifying the Routing Table Construction Mechanism", Published in IEEE International Conference on Reliability, Optimization and Information Technology, pp. 182-187, Feb. 2014.

[32] Fotohi R, Jamali S, Sarkohaki F., "Performance Evaluation of AODV, LHC-AODV, OLSR, UL-OLSR, DSDV Routing Protocols", Published online in MECS International Journal of Information Technology and Computer Science IJITCS, DOI: 10.5815/ijitcs.2013.10.03, pp. 21-29, September 2013.

[33] Shahram Behzad, Reza Fotohi and Shahram Jamali, "Improvement over the OLSR Routing Protocol in Mobile Ad Hoc Networks by Eliminating the Unnecessary Loops", Published online in MECS I.J. of Information Technology and Computer Science, DOI: 10.5815/ijitcs.2013.06.03, pp. 16-22, May 2013.

\section{Authors' Profiles}

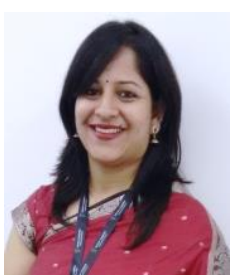

Shelja Sharma: Currently pursuing Doctoral Research in the area "Mobile AdHoc Networks" from Manav Rachna International Institute of Research and Studies (formerly MRIU), Faridabad, India. She has received a Bachelor's of Engineering degree in Computer Science \& Engineering with Honors from Rajasthan University, Jaipur, India in 2005 and a Masters of Technology degree in Computer Engineering from MRIU, Faridabad, India in 2011.

Presently, she is working as an Assistant Professor in the Department of Computer Science \& Engineering, Manav Rachna International Institute of Research and Studies 
(formerly MRIU), Faridabad, India. She has eleven years of total experience and published eight Research publications in International Journals and International conferences. Her primary research area includes "Mobile Ad-Hoc Networks".
His areas of interest include Ad-hoc Networks, Database Management System and Data Mining.

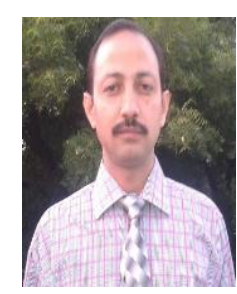

Dr. Suresh Kumar: Currently working as a Professor, in the Department of Computer Science \& Engineering, Manav Rachna International Institute of Research and Studies (formerly MRIU), Faridabad, India. $\mathrm{He}$ has more than seventeen years of experience and more than forty-five research publications in diversified areas.

How to cite this paper: Shelja Sharma, Suresh Kumar,"Simulation Analysis of OLSR and Its Variant with Cooperative MPR Selection on NS-2.35 in Mobile Ad-Hoc Networks", International Journal of Computer Network and Information Security(IJCNIS), Vol.10, No.7, pp.44-51, 2018.DOI: 10.5815/ijcnis.2018.07.05 